\title{
High Oxygen Concentrations Adversely Affect the Performance of Pulmonary Surfactant
}

\author{
Craig D Smallwood RRT, Parnian Boloori-Zadeh PhD, Maricris R Silva PhD, and \\ Andrew Gouldstone PhD
}

\begin{abstract}
BACKGROUND: Although effective in the neonatal population, exogenous pulmonary surfactant has not demonstrated a benefit in pediatric and adult subjects with hypoxic lung injury despite a sound physiologic rationale. Importantly, neonatal surfactant replacement therapy is administered in conjunction with low fractional $\mathrm{F}_{\mathrm{IO}_{2}}$ while pediatric/adult therapy is administered with high $\mathrm{F}_{\mathrm{IO}_{2}}$. We suspected a connection between $\mathrm{F}_{\mathrm{IO}_{2}}$ and surfactant performance. Therefore, we sought to assess a possible mechanism by which the activity of pulmonary surfactant is adversely affected by direct oxygen exposure in in vitro experiments. METHODS: The mechanical performance of pulmonary surfactant was evaluated using 2 methods. First, Langmuir-Wilhelmy balance was utilized to study the reduction in surface area $(\delta \mathrm{A})$ of surfactant to achieve a low bound value of surface tension after repeated compression and expansion cycles. Second, dynamic light scattering was utilized to measure the size of pulmonary surfactant particles in aqueous suspension. For both experiments, comparisons were made between surfactant exposed to $21 \%$ and $100 \%$ oxygen. RESULTS: The $\delta \mathrm{A}$ of surfactant was $21.1 \pm 2.0 \%$ and $35.8 \pm 2.0 \%$ during exposure to $21 \%$ and $100 \%$ oxygen, respectively $(P=.02)$. Furthermore, dynamic light-scattering experiments revealed a micelle diameter of $336.0 \pm 12.5 \mu \mathrm{m}$ and $280.2 \pm 11.0 \mu \mathrm{m}$ in $21 \%$ and $100 \%$ oxygen, respectively $(P<.001)$, corresponding to a $\sim 16 \%$ decrease in micelle diameter following exposure to $100 \%$ oxygen. CONCLUSIONS: The characteristics of pulmonary surfactant were adversely affected by short-term exposure to oxygen. Specifically, surface tension studies revealed that short-term exposure of surfactant film to high concentrations of oxygen expedited the frangibility of pulmonary surfactant, as shown with the $\delta \mathrm{A}$. This suggests that reductions in pulmonary compliance and associated adverse effects could begin to take effect in a very short period of time. If these findings can be demonstrated in vivo, a role for reduced $\mathrm{F}_{\mathrm{IO}_{2}}$ during exogenous surfactant delivery may have a clinical benefit. Key words: pulmonary surfactant; oxygen toxicity; infasurf; langmuir-wilhelmy; dynamic light scattering. [Respir Care 2017;62(8):1085-1090. (c) 2017 Daedalus Enterprises]
\end{abstract}

\section{Introduction}

Human metabolism relies primarily on an aerobic process for the combustion of nutrients and extraction of energy at the cellular level. The process consumes oxygen and generates

\footnotetext{
Mr Smallwood and Drs Boloori-Zaden, Silva and Gouldstone are affiliated with the College of Engineering, Northeastern University; and $\mathrm{Mr}$ Smallwood also is affiliated with the Division of Critical Care Medicine, Department of Anesthesia, Perioperative and Pain Medicine, Boston Children's Hospital, and Harvard Medical School, Boston, Massachusetts.
}

The authors have disclosed no conflicts of interest.

Mr Smallwood and Dr Boloori-Zadeh contributed equally to this work. carbon dioxide. In healthy subjects, ambient concentrations of oxygen $(21 \%)$ are sufficient to sustain metabolism. However, a number of pathophysiologic processes can impede exchange of oxygen from the lungs into the blood, and therefore administration of high concentrations of oxygen is required to maintain adequate arterial oxygen levels and tissue oxygenation. Although sometimes clinically indicated, high

\footnotetext{
Correspondence: Andrew Gouldstone PhD, College of Engineering, Department of Mechanical and Industrial Engineering, Northeastern University, Boston, MA 02115. E-mail: a.gouldstone@ northeastern.edu.
}

DOI: $10.4187 /$ respcare. 05388 
oxygen concentrations may lead to oxygen toxicity. In vivo research suggests this toxicity may manifest as changes in airways responsiveness, inflammation, increased lung volumes, and depletion of type II pneumocyte proliferation. ${ }^{1-3}$

Pulmonary surfactant has been the subject of extensive research since its successful administration in newborn infants with respiratory distress syndrome was described by Fujiwara et $\mathrm{al}^{4}$ in the early 1980s. Since that time, its role in the care of premature infants as well as chemical constituents and specific function have been well described. ${ }^{5}$ More recently, the role of exogenous surfactant in the care of adult and pediatric subjects with ARDS has been investigated.6-9 Pathogenesis of ARDS has been partly attributed to alterations in endogenous surfactant. ${ }^{10}$ The physiologic rationale for the administration of surfactant in this population is to restore surfactant function, thereby improving pulmonary system compliance and oxygenation. Despite promising data in pilot trials, large controlled investigations have not demonstrated a clear benefit of exogenous surfactant in subjects with ARDS as it had little or no effect on oxygenation or long-term outcomes. ${ }^{7,11}$ However, since the majority of exogenous surfactant protocols in pediatric and adult patients call for high concentrations of inspired oxygen during therapy (which is not the case in the neonatal population), we suspected that there may be a connection between oxygen concentration and surfactant performance.

Furthermore, most research has focused on the cellular mechanisms of oxygen toxicity, but the effects of direct high-concentration oxygen on surfactant mechanical properties have not been adequately described in a controlled laboratory investigation. Oxygen toxicity, which results from the delivery of increased concentrations of oxygen, has been associated with absorption atelectasis, pulmonary edema and lung fibrosis. ${ }^{12-15}$ Oxygen toxicity is compounded by increased $\mathrm{F}_{\mathrm{IO}_{2}}$ as well as duration of exposure. Typically, $\mathrm{F}_{\mathrm{IO}_{2}} \leq 0.50$ has been recommended as a therapeutic target to reduce the likelihood of oxygen toxicity in human subjects. ${ }^{16}$

A biochemical mechanism, as the basis for oxygen toxicity, has been proposed; it results from the cellular production of partly reduced oxygen metabolites. ${ }^{17,18}$ Indeed, lung injury may be exacerbated by an oxygen free radical process that results in cellular damage and impeded gas exchange. ${ }^{19-22}$ In vivo translational research has demonstrated that administration of an oxygen free radical-fighting molecule, superoxide dismutase, prevents many deleterious effects of breathing high-concentration oxygen in an animal model. ${ }^{23}$ However, survival rates, morphologic changes, and histologic findings were not completely preserved, suggesting mechanisms other than those mediated by oxygen free radicals may contribute to lung injury.

The present research group has previously demonstrated that in excised lungs, exposure to different oxygen concentrations can have near-immediate effects on mechani-

\section{QUICK LOOK}

\section{Current knowledge}

Exogenous surfactant administration is effective in the neonatal population with surfactant deficiency, but is not effective for pediatric and adult patients with ARDS despite data showing that surfactant function is allayed. We suspected a connection between oxygen concentration and surfactant performance.

\section{What this paper contributes to our knowledge}

The characteristics of pulmonary surfactant were adversely affected by short-term exposure to oxygen. Specifically, surface tension studies revealed that shortterm exposure of surfactant film to high concentrations of oxygen expedited the frangibility of pulmonary surfactant, as shown with the surface area change. These data suggest that reductions in pulmonary compliance and associated adverse effects could begin to take effect in a very short period of time.

cal behavior. That is to say, lungs inflated with $100 \%$ oxygen have significantly greater stiffness and hysteresis than those exposed to air. This effect was most apparent at low lung pressures $\left(\sim 4 \mathrm{~cm} \mathrm{H}_{2} \mathrm{O}\right)$ and smaller at much higher pressures $\left(\sim 20 \mathrm{~cm} \mathrm{H}_{2} \mathrm{O}\right) .{ }^{24}$ Mechanical responses at low pressures are known to be dominated by surfactant behavior, and we hypothesized that the gas composition was directly affecting this substance.

It should be noted that experiments by Wildebeer-Ven$\mathrm{ema}^{25}$ showed that exposure to different gases had little to no effect on the static minimum surface tension of surfactant. However, that study did not explore dynamic surface tension and re-spreading of surfactant, which is very important in vivo because the surface area expands and contracts inside the alveoli with breathing. Therefore, we sought to describe the performance of isolated pulmonary surfactant at high oxygen concentration (100\%) and low oxygen concentration (air, 21\%) through the careful implementation of 2 distinct experiments: 1) on the performance (directly related to ability of surfactant to redistribute), and 2) on the suspension structure of surfactant.

\section{Methods}

\section{Surfactant Preparation}

The surfactant preparation utilized for the experiments was Infasurf (ONY, Amherst, New York), a bovine, nonpyrogenic pulmonary surfactant. It is a natural calf lung surfactant extract that includes phospholipids, mainly di- 
palmitoylphosphatidylcholine, neutral lipids, and hydrophobic surfactant-associated proteins $\mathrm{B}$ and $\mathrm{C}$, and is cleared for human use by the FDA. According to established laboratory protocol, the surfactant was suspended in chloroform-anhydrous (Sigma-Aldrich, St. Louis, Missouri) to a concentration of $1 \mathrm{mg} / \mathrm{mL}$ and stored in a temperature-regulated refrigerator $\left(\sim 7^{\circ} \mathrm{C}\right)$.

\section{Surface Tension Measurement}

Surface tension studies were performed using a Langmuir-Wilhelmy balance (Micro Trough X, Kibron, Helsinki, Finland) with a surface area of $\sim 125 \mathrm{~cm}^{2}$. The technique has been used previously to characterize pulmonary surfactant in vitro. ${ }^{26,27}$ Fundamentally, the balance is used to quantify surface tension through the ability of the surfactant to redistribute across the surface area of the device after changing the surface area. This property is associated with substance frangibility, and in the context of surfactant, its ability to reduce surface tension and increase compliance in vivo. ${ }^{27}$ Preceding the experiments, the equipment was calibrated according to the manufac-

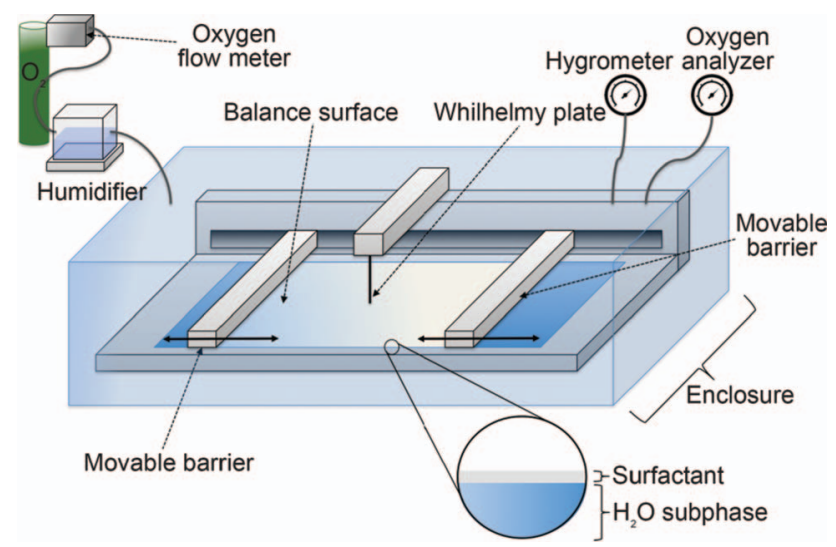

Fig. 1. Illustration of experimental setup with the Langmuir-Wilhelmy balance. turer's specifications. Preparation of the instrument included deposition of a $50 \mu \mathrm{L}$ of pure water onto the balance plate using a micro-syringe. The water layer serves as the subphase, upon which the surfactant is deposited. After being warmed to ambient temperature, $20 \mu \mathrm{L}$ of surfactant was deposited on the water subphase. An illustration of the experimental setup is shown in Figure 1. The entire device was enclosed in a humidity- and temperature-controlled environmental enclosure (depicted in Fig. 1). The relative humidity was maintained at $\sim 80 \%$ and room temperature $\left(\sim 21^{\circ} \mathrm{C}\right)$. The surfactant surface was repeatedly compressed with the device (ie, cycled). The device was cycled between lower and upper bounds of surface tension $\gamma$ : $15 \mathrm{mN} / \mathrm{m}$ and $45 \mathrm{mN} / \mathrm{m}$ respectively, at $93.5 \mathrm{~mm} / \mathrm{min}$ (this corresponds to a nominal $50 \%$ area change/min). After a typical Langmuir-Wilhelmy cycle (see Fig. 2), the $\gamma$-area curve shifts to the left; this is because surfactant molecules are lost to the aqueous subphase, and thus slightly greater compression is required to achieve the programmed lower bound of $\gamma$. Our experiments aimed to measure the effect of oxygen concentration on this shift. Two distinct experimental procedures were conducted: in air, and in $100 \%$ oxygen. To introduce oxygen into the system, pure oxygen was introduced into the enclosure at a flow of $3 \mathrm{~L} / \mathrm{min}$. The oxygen concentration was continuously monitored during the experiments with the Oxygen-Meter 200 (Apogee Instruments, Logan, Utah). Each experiment comprised 30 cycles. A total of 3 experiments were conducted in $21 \%$ oxygen, and 6 in $100 \%$ oxygen. Surface tension data were continuously recorded utilizing FilmWare (version 3.57, Kibron). In summary, 2 distinct experiments were conducted on surfactant: 1) in air, and 2) in $100 \%$ oxygen.

\section{Calculation of Difference in Surface Area}

For each 30-cycle experiment, the aforementioned shift (expressed as a percentage, $\delta \mathrm{A}$ ) was calculated from the first to last cycle. A smaller $\delta \mathrm{A}$ indicates a greater ability
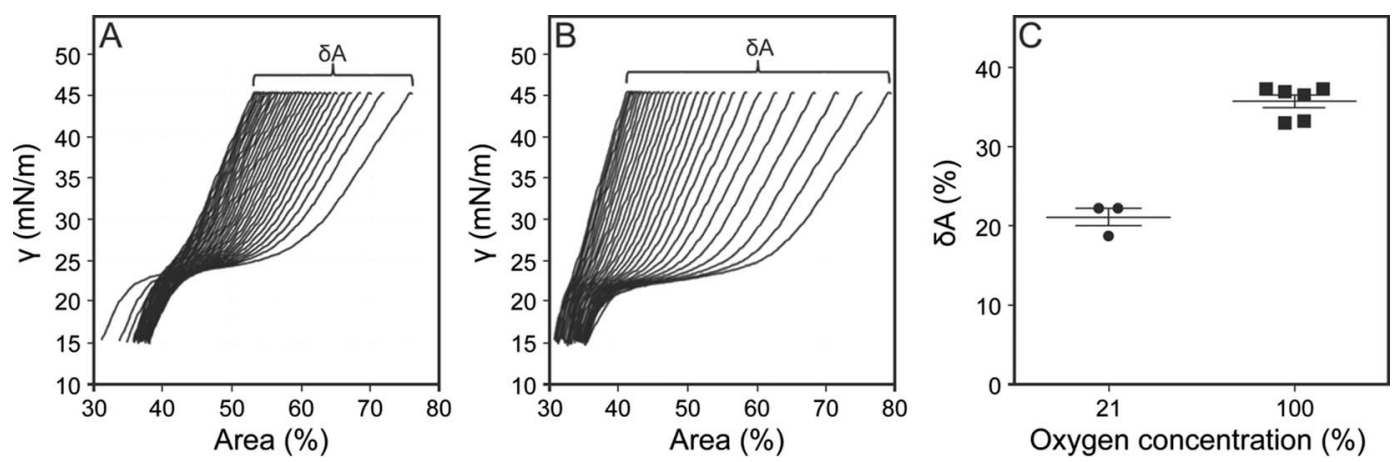

Fig. 2. Plot of the surface tension $(\gamma)$ and area. The area is the surface area that the surfactant occupied on the Langmuir-Wilhelmy balance (expressed as a percentage of total) in A: $21 \%$ oxygen and B: $100 \%$ oxygen. C: Plot of surface area change $(\delta \mathrm{A})$ between $21 \%$ and $100 \%$ oxygen. Horizontal lines represent the mean and whiskers denote \pm SD. $\delta A$ is the change in area expressed as a percentage. 
of surfactant to dynamically redistribute. An illustration of the calculation of the $\delta \mathrm{A}$ is shown in Figure 2.

\section{Surfactant Micelle Size Measurement Using Dynamic Light Scattering}

A surfactant suspension was prepared with water in 50$\mathrm{mL}$ glass beakers at a ratio of $3 \mu \mathrm{L}$ surfactant to $2 \mathrm{~mL}$ water. The preparation was mixed using a magnetic stirrer, according to standard laboratory protocol. The use of a magnetic stirrer ensures that the preparation is thoroughly but gently mixed without disturbing the surfactant to the point of foaming. Four iterations were performed: 1) surfactant suspension and introduction of $2 \mathrm{~L} / \mathrm{min}$ air, 2) surfactant suspension and introduction of $2 \mathrm{~L} / \mathrm{min} 100 \%$ oxygen, 3) water-only control and $2 \mathrm{~L} / \mathrm{min}$ air, and 4) wateronly control and $2 \mathrm{~L} / \mathrm{min} 100 \%$ oxygen. In all cases, the gases were introduced for a total of $3 \mathrm{~h}$. Each beaker was then emptied into a measurement cuvette. The surfactant particles formed varying-sized micelles. The size of these micelles was measured using a dynamic light-scattering device (NanoBrook 90 Plus, Brookhaven Instruments, Holtsville, New York). Dynamic light scattering is a technique used to quantify the size of small particles in suspension, and it has been used widely in chemistry and biology. ${ }^{28,29}$ Measurements were repeated 10 times for each the air and the $100 \%$ oxygen preparation.

\section{Statistical Analyses}

Data were imported into Excel (version 14.6.7, Microsoft, Redmond, Washington) and Prism (version 6.0g, GraphPad Software, San Diego, California). The $\delta$ A of surfactant in air and oxygen were compared using a MannWhitney $\mathrm{U}$ test. The measured micelle size data (from dynamic light scattering) were compared using an unpaired $t$ test.

\section{Results}

\section{Surface Tension Measurement}

We observed a statistically significant difference between the $\delta \mathrm{A}$ of surfactant during exposure to $21 \%$ oxygen and $100 \%$ oxygen $(21.1 \pm 2.0 \%$ vs $35.8 \pm 2.0 \%, P=.02)$ (see Fig. 2). Given the fact, that, in oxygen, a greater surface area change is required to achieve the target lower surface tension, more surfactant is lost to the aqueous subphase, and we attribute that to increased frangibility.

\section{Surfactant Micelle Diameter Measurement Using Dynamic Light Scattering}

Dynamic light-scattering experiments revealed a micelle diameter of $336.0 \pm 12.5 \mu \mathrm{m}$ and $280.2 \pm 11.0 \mu \mathrm{m}$ in
$21 \%$ and $100 \%$ oxygen, respectively $(P<.001)$; corresponding to a $\sim 16 \%$ decrease in micelle diameter following exposure to $100 \%$ oxygen (Fig. 3 ).

\section{Discussion}

In an in vitro model, we demonstrated a statistically significant difference in the area change during controlled cycling of pulmonary surfactant, suggesting that pulmonary surfactant performance is negatively affected by exposure to high oxygen concentrations. As depicted in Figure 2, when surfactant is exposed to pure oxygen, a more pronounced reduction in surface area necessary to achieve a lower surface tension $\gamma$ is observed. This indicates a lower surface density of available surfactant molecules on the aqueous surface. This occurs as surfactant molecules are lost within the aqueous subphase following repeated cycling in oxygen. It is conceivable that this loss is because of increased frangibility of the surfactant in $100 \%$ oxygen.

Under compression, molecule chains are driven into the aqueous subphase and curl because of the hydrophobic and hydrophilic nature of the surfactant molecules. Ideally, this curl would be tolerated and upon re-expansion, a majority of molecules would return to the surface. However, at a particular limit, the curled portion breaks off and forms a micelle (Fig. 3). We hypothesize that $100 \%$ oxygen reduces this limit, and allows the surfactant to break off more easily under compression. This assertion is consistent with our results from dynamic light scattering, where statistically significant differences in micelle diameter were observed. The smaller suspended particles (observed in $100 \%$ oxygen) would result from a more frangible surfactant.

Importantly, our data suggest that pulmonary surfactant performance is negatively affected by exposure to high oxygen concentrations. Surfactant supplementation has not been shown to produce a sustained or significant positive impact in patients with hypoxic lung injury. ${ }^{7}$ The results of the present work demonstrate the rapid deterioration in mechanical performance of surfactant as a result of high $\mathrm{O}_{2}$ exposure. This may offer a partial explanation as to why supplemental surfactant has not produced significant improvements in oxygenation and long-term outcomes in patients with lung injury.

There are important limitations to the present work that should be noted. First, instead of human derived surfactant, isolated bovine surfactant was used. However, bovine surfactant is analogous to human pulmonary surfactant and is approved in many countries for surfactant replacement therapy. Second, the environmental factors were not identical to those found within the lungs. The equipment and experimental conditions precluded exact temperature and humidity, but efforts were made to ensure that humidity was added to the environment. Preliminary experiments 

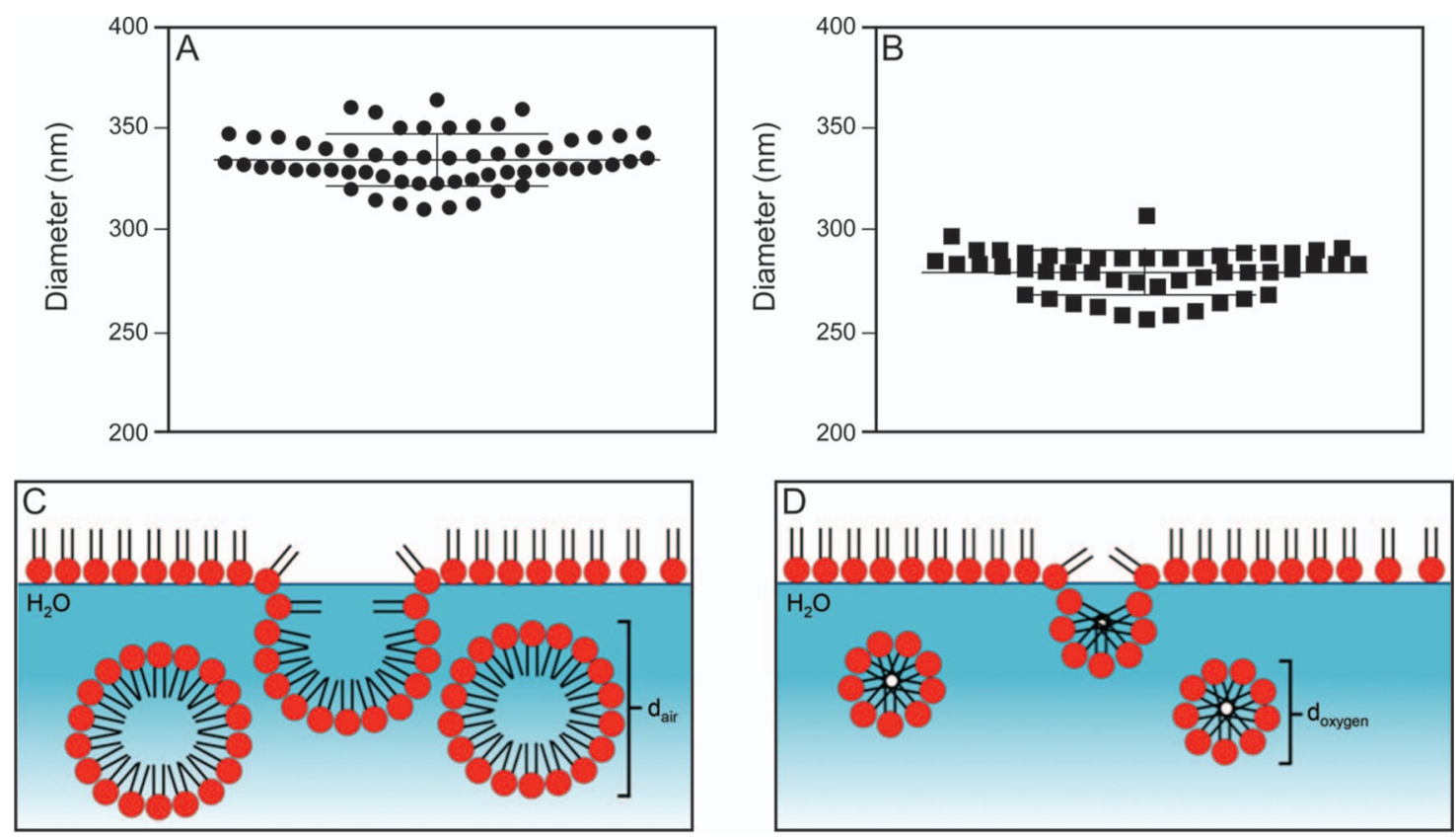

Fig. 3. Results from the dynamic light-scattering experiments. The diameter of the surfactant micelles from each sample are shown in A: $21 \%$ oxygen, and B: $100 \%$ oxygen. Micelle diameter was significantly smaller in $100 \%$ oxygen vs $21 \%$ oxygen $(280.2 \pm 11.0 \mu \mathrm{m}$ vs $336.0 \pm 12.5 \mu \mathrm{m}, P<.001$.). Horizontal bars represent the mean and whiskers show the SD of the entire sample. In $\mathrm{C}(21 \%$ oxygen) and D (100\% oxygen), graphic depictions of the micelle diameter are shown to illustrate the measurements (surfactant particle size not drawn to scale, for illustration only).

with surfactant with the equipment revealed that it evaporated at temperatures $\sim 37^{\circ} \mathrm{C}$, which would significantly confound the surface tension and area measurements, and we therefore decided to limit the temperature to reduce this risk. Third, the colloid preparation utilized deionized water and not saline. However, since the protocol followed precedent, it's unlikely that this would have a significant confounding effect on the results. Fourth, our experiment was designed such that only $2 \mathrm{~F}_{\mathrm{IO}_{2}}$ concentrations were investigated. This limited our ability to determine if a critical threshold for $\mathrm{F}_{\mathrm{IO}_{2}}$ exists below a $\mathrm{F}_{\mathrm{IO}_{2}}$ of 1.0. In the future, it will be important to investigate a range of $\mathrm{F}_{\mathrm{IO}_{2}}$ to assess whether a concentration-dependent adverse effect on surfactant exists. Last, all experiments were conducted in vitro. However, surface tension and micelle diameter measurements are not possible to obtain in vivo with presently available equipment.

It is important to note that exogenous surfactant has been targeted in conditions with distinct underlying pathophysiologic processes. In terms of surfactant function, respiratory distress syndrome in neonatal patients can be characterized as a surfactant deficiency and acute lung injury, or ARDS can be characterized as a surfactant dysfunction. ${ }^{30-33}$ Factors such as airway edema, fibrosis, infection, and extrinsic airway pressures may contribute to surfactant dysfunction during ARDS, but could not be included in the present model. Since it is outside the scope of the present work, we cannot speculate on what the compounded effect of high-flow oxygen therapy and other factors would be upon surfactant mechanics, and further work is indicated in this area. Interestingly, neonatal patients typically receive lower $\mathrm{F}_{\mathrm{IO}_{2}}(0.21-0.30)$ as a function of targeting a low $\mathrm{S}_{\mathrm{pO}_{2}}$ for the prevention of retinopathy of prematurity as well as preventing development of bronchopulmonary dysplasia, whereas pediatric and adult patients with acute lung injury may receive $\mathrm{F}_{\mathrm{IO}_{2}}, 0.4-1.0$, particularly around times associated with care..$^{34,35}$ Despite the differences in pathophysiology, we questioned whether the $\mathrm{F}_{\mathrm{IO}_{2}}$ around the time of surfactant administration could affect the properties of surfactant, which, in turn could manifest as important clinical differences. Our data do indeed suggest a deterioration of surfactant function in high-oxygen concentration environments as evidenced by the increased frangibility of the molecules.

Future research should seek to assess the reversibility of oxygen's adverse effect on pulmonary surfactant through in vitro studies. Further, since we tested only 2 distinct oxygen concentrations, a specific concentration at which the negative effects of oxygen are not observed is not known. Work that assesses surfactant function over a wide range of oxygen concentrations may help to identify a specific level, below which surfactant is only minimally adversely affected. Further, in vivo work assessing the combined effects of factors known to cause surfactant dysfunction and induce lung injury with high oxygen concentrations should be completed. 


\section{High $\mathrm{F}_{\mathrm{IO}_{2}}$ And Pulmonary Surfactant Performance}

\section{Conclusions}

Surface tension studies revealed that short-term exposure of surfactant film to high concentrations of oxygen expedited the frangibility of pulmonary surfactant, as shown with the altered surface area required to achieve a minimum surface tension value. This interpretation was consistent with mechanistically distinct light-scattering experiments on aqueous surfactant suspensions. This may suggest that reductions in pulmonary compliance and associated adverse effects could begin to take effect in a very short period of time. If these findings can be demonstrated in vivo, then a role for reduced $\mathrm{F}_{\mathrm{IO}_{2}}$ during exogenous surfactant delivery may be indicated and could enhance clinical benefit.

\section{ACKNOWLEDGMENTS}

The authors acknowledge Dr Heather Clark of Pharmaceutical Sciences at Northeastern University for usage of the light scattering equipment, as well as Fulden Buyukozturk, Selena Di Maio, and Dr Rebecca Carrier for helpful guidance on the light scattering experiments.

\section{REFERENCES}

1. Denis D, Fayon MJ, Berger P, Molimard M, De Lara MT, Roux E, Marthan R. Prolonged moderate hyperoxia induces hyperresponsiveness and airway inflammation in newborn rats. Pediatr Res 2001; 50(4):515-519.

2. Dauger S, Ferkdadji L, Saumon G, Vardon G, Peuchmaur M, Gaultier C, Gallego J. Neonatal exposure to $65 \%$ oxygen durably impairs lung architecture and breathing pattern in adult mice. Chest 2003; 123(2):530-538.

3. Yee M, Vitiello PF, Roper JM, Staversky RJ, Wright TW, McGrathMorrow SA, et al. Type II epithelial cells are critical target for hyperoxia-mediated impairment of postnatal lung development. Am J Physiol Lung Cell Mol Physiol 2006;291(5):L1101-L1111.

4. Fujiwara T, Maeta H, Chida S, Morita T, Watabe Y, Abe T. Artificial surfactant therapy in hyaline-membrane disease. Lancet 1980; 1(8159):55-59.

5. Logan JW, Moya FR. Animal-derived surfactants for the treatment and prevention of neonatal respiratory distress syndrome: summary of clinical trials. Ther Clin Risk Manag 2009;5(1):251-260.

6. Willson DF, Thomas NJ, Tamburro R, Truemper E, Truwit J, Conaway $\mathrm{M}$, et al. Pediatric calfactant in acute respiratory distress syndrome trial. Pediatr Crit Care Med 2013;14(7):657-665.

7. Willson DF, Truwit JD, Conaway MR, Traul CS, Egan EE. The adult calfactant in acute respiratory distress syndrome trial. Chest 2015; 148(2):356-364

8. Willson DF, Thomas NJ, Markovitz BP, Bauman LA, DiCarlo JV, Pon $\mathrm{S}$, et al. Effect of exogenous surfactant (calfactant) in pediatric acute lung injury: a randomized controlled trial. JAMA 2005;293(4):470-476.

9. Duffett M, Choong K, Ng V, Randolph A, Cook DJ. Surfactant therapy for acute respiratory failure in children: a systematic review and meta-analysis. Crit Care 2007;11(3):R66.

10. Royall JA, Levin DL. Adult respiratory distress syndrome in pediatric patients. I. Clinical aspects, pathophysiology, pathology, and mechanisms of lung injury. J Pediatr 1988;112(2):169-180.

11. Kesecioglu J, Beale R, Stewart TE, Findlay GP, Rouby JJ, Holzapfel $\mathrm{L}$, et al. Exogenous natural surfactant for treatment of acute lung injury and the acute respiratory distress syndrome. Am J Respir Crit Care Med 2009;180(10):989-994

12. Gore A, Muralidhar M, Espey MG, Degenhardt K, Mantell LL. Hyperoxia sensing: from molecular mechanisms to significance in disease. J Immunotoxicol 2010;7(4):239-254.

13. Nunn JF. Conscious volunteers developed hypoxemia and pulmonary collapse when breathing air and oxygen at reduced lung volume. Anesthesiology 2003;98(1):258-259.

14. Thomson L, Paton J. Oxygen toxicity. Paediatr Respir Rev 2014; 15(2):120-123

15. Kallet RH, Matthay MA. Hyperoxic acute lung injury. Respir Care 2013;58(1):123-141.

16. Jackson RM. Pulmonary oxygen toxicity. Chest 1985;88(6):900-905.

17. Fridovich I. The biology of oxygen radicals. Science 1978;201(4359): 875-880.

18. McCord JM, Fridovich I. The biology and pathology of oxygen radicals. Ann Intern Med 1978;89(1):122-127.

19. Tate RM, Repine JE. Neutrophils and the adult respiratory distress syndrome. Am Rev Respir Dis 1983;128(3):552-559.

20. Crapo JD. Morphologic changes in pulmonary oxygen toxicity. Annu Rev Physiol 1986;48:721-731.

21. Saugstad OD. Bronchopulmonary dysplasia-oxidative stress and antioxidants. Semin Neonatol 2003;8(1):39-49.

22. Pacher P, Beckman JS, Liaudet L. Nitric oxide and peroxynitrite in health and disease. Physiol Rev 2007;87(1):315-424.

23. Yen CC, Lai YW, Chen HL, Lai CW, Lin CY, Chen W, et al. Aerosolized human extracellular superoxide dismutase prevents hyperoxia-induced lung injury. PLoS One 2011;6(10):e26870.

24. Silva V, Grande AJ, Rech CR, Peccin MS. Geoprocessing via google maps for assessing obesogenic built environments related to physical activity and chronic noncommunicable diseases: validity and reliability. J Healthc Eng 2015;6(1):41-54.

25. Wildeboer-Venema F. Influence of nitrogen, oxygen, air and alveolar gas upon surface tension of lung surfactant. Respir Physiol 1984;58(1):1-14.

26. Keating E, Rahman L, Francis J, Petersen A, Possmayer F, Veldhuizen R, Petersen NO. Effect of cholesterol on the biophysical and physiological properties of a clinical pulmonary surfactant. Biophys J 2007;93(4):1391-1401.

27. Schürch S. Surface tension at low lung volumes: dependence on time and alveolar size. Respir Physiol 1982;48(3):339-355.

28. Berne BJ, Pecora R. Dynamic light scattering: with applications to chemistry, biology, and physics. Mineola, N.Y.: Dover Publications; 2000.

29. Wax A, Backman V. Biomedical applications of light scattering. New York: McGraw-Hill; 2010.

30. Lewis JF, Ikegami M, Jobe AH. Altered surfactant function and metabolism in rabbits with acute lung injury. J Appl Physiol (1985) 1990;69(6):2303-2310.

31. Veldhuizen RA, McCaig LA, Akino T, Lewis JF. Pulmonary surfactant subfractions in patients with the acute respiratory distress syndrome. Am J Respir Crit Care Med 1995;152(6 Pt 1):1867-1871.

32. Günther A, Siebert C, Schmidt R, Ziegler S, Grimminger F, Yabut $\mathrm{M}$, et al. Surfactant alterations in severe pneumonia, acute respiratory distress syndrome, and cardiogenic lung edema. Am J Respir Crit Care Med 1996;153(1):176-184.

33. Ardell S, Pfister RH, Soll R. Animal derived surfactant extract versus protein free synthetic surfactant for the prevention and treatment of respiratory distress syndrome. Cochrane Database Syst Rev 2015(5):CD000144.

34. Wyllie J, Perlman JM, Kattwinkel J, Wyckoff MH, Aziz K, Guinsburg R, et al. Part 7: Neonatal resuscitation: 2015 international consensus on cardiopulmonary resuscitation and emergency cardiovascular care science with treatment recommendations. Resuscitation 2015;95:e169-201.

35. Laughon M, Allred EN, Bose C, O'Shea TM, Van Marter LJ, Ehrenkranz RA, et al. Patterns of respiratory disease during the first 2 postnatal weeks in extremely premature infants. Pediatrics 2009;123(4):1124-1131. 